Case Study

\title{
Cenesthopathy-The Paradigm in Schizophrenia
}

Anum Haider

AQ Khan Center, Institute of Behavioral Sciences Karachi

Corresponding Author: dranum.haider@gmail.com

\section{Abstract}

In this article, the frequently presented but underestimated or neglected area has been unveiled. The Abnormal body sensations, Cenesthesias has been discussed in the context of schizophrenia. The identified underlying Psychophysiological mechanisms and treatment options will also be discussed along with future recommendations. Two cases will be presented to contemplate this condition.

\section{Keywords}

Cenesthopathic, Schizophrenia, abnormal body sensations, prognosis

\section{Introduction}

The term cenesthopathy coined by Dupre and Camus in 1907 and described it as a phenomenology in mental illness (Takahash et al., 2013). This term has been derived from "cenethesia" which means abnormal body sensations. The abnormal body sensations come as frequent complaint in Schizophrenia (Jenkins et al., 2007).Huber in 1957 proposed cenesthopathic schizophrenia as "a subtype of Schizophrenia in which bizarre peculiar abnormal bodily sensations dominate, these are difficult to describe, chronic insidious in course and with limited psychotic symptoms" (Takahash, et al., 2013). It not only presents a separate entity of Schizophrenia but can also be present as onset and prodromal of Schizophrenia (Stanghellini, et al., 2014).Its prevalence as a separate entity is $6.25-23.3 \%$ (Chang, 2014). It may appear as an onset of illness in $40 \%$ of cases while the rate of change in form of illness in later course is $75.7 \%$ (Rajendar, et al., 2010). In this category of Schizophrenia, tactile hallucinations and somatosensory delusional beliefs are common (Blom, 2012). The most common presentations are dysmorphic or pain like experiences, morbid objectification or devitalization, dynamization of bodily boundaries and construction (Stanghellini, et al., 2014).

The suggested underpinning neurobiological and psychophysiological mechanisms are central brain atrophy, thalamic disruption, cortical disorganization, alteration in pain perception and modulation (i.e. noxious inhibitory control and excitatory endogenous pain mechanisms). These changes if matches with schizophrenic pathophysiological factors like genetic vulnerability neurochemical changes-decreased inhibitory GABAergic and Increased excitatory Glutamatergic activities, NMDA receptor hypo function, changes in dopaminergic and cholinergic transmission can result in cenesthopathic entity of Schizophrenia spectrum disorder (Takahash, et al., 2013, Chang, et al., 2014). Cenesthopathy can present with both psychiatric and organic disorders (Dementia). As a Psychiatric disorder it can be a sole presentation or a symptom of paranoid psychosis (Takahash, et al., 2013 \& Chang, 2014). Despite of such frequent and even dominant presentation, it could not get adequate importance (Stanghellini, et al., 2014). Moreover the term cenesthopathic schizophrenia has not been introduced in any classification system.

\section{Anum Haider}


However in ICD-10 it can be classified in the category of other Schizophrenic disorders (Jenkins, et al., 2007).It is a very important diagnosis to be aware of because it poses great burden of disability as a result of negative symptoms and personality deterioration (Chang, 2014). Early recognition and start of treatment is crucial for good prognosis and limiting the disability (Stanghellini, et al., 2014 \& Rohircht, et al., 2010).

The aim of presentation of following two cases is to increase the awareness of the problem and to promote early recognition and better prognosis.

\section{CASE-1}

Mr. Y 60years old married male, father of three children, retired, educated up to intermediate, follows Muslim religion and belongs to Asian ethnicity. He has no known comorbid, presented by daughter in psychiatric clinic with complaints of; Excessive hand washing and feeling of sticky secretions from body for more than one year. The illness started with somatic complaints such as difficulty in urination and other gut related symptoms. Those had been investigated with unremarkable findings and ultimately handled by different physicians as medically unexplained symptoms. Then gradually the illness presented differently by patient with excessive hand washing and preoccupation with body because of the release of sticky secretions from skin including the mouth cavity and ears. The patient was convinced and claimed that he could see those secretions releasing from the skin in almost entire body but most at the conspicuous parts. He used to wash excessively the alleged affected areas and kept Vaseline all time with him to apply at the dry skin after washing. He reduced to take food due to those secretions and consequently his body weight was reduced.
His sleep was disturbed and sociooccupational functioning was ceased completely. On Mental State Examination; there was emotional bluntness, physical agitation, scanty speech, refusal of auditory hallucinations. Somatic delusion/ hallucination (Fully convicted with the secretions and able to see it) Preoccupation with the secretions and interested to get rid of it. Absence of insight and grossly sound cognitive functions. Physical examination and laboratory investigation unremarkable. Treatment started with second generation antipsychotics (tab olanzapine 10mg) and called for follow up.

\section{CASE-2}

Mrs. X, 55years old divorced household lady educated up to graduation, follows Muslim religion, belongs to Asian ethnicity. She has no known comorbid, presented by brother in psychiatric clinic with complains of; feeling of discharge and malodor from body (including genitalia), avoidance of religious activities. These were insidious in onset and aggravated for past few months. The illness started gradually with social isolation and altered behavior (self-neglect and reduced oral intake). It then became obvious when she clearly started complaining about body secretions and behaving accordingly (excessive hand washing and securing from paravaginal discharge) and as well as asking family members if they could smell the bad odor from her body. She abandoned offering prayers contemplating herself contaminated. She also stopped socialization and started talking limitedly. She argued with baseless justifications on confrontation of family members. On Mental state examination; she had found with blunt affect, irritable on skepticism, preoccupied with body odor and discharge, poor rapport, convicted with her beliefs (somatic delusion and hallucination), refusing auditory hallucinations, lack of 
insight and intact gross cognitive functioning. Gynecologist cleared her from any pathology. Physical examination and labs were also unremarkable. Treatment started with Tablet Olanzapine10mg and sertraline $50 \mathrm{mg}$ and referred for psychological evaluation and therapy. Family Psychoeducational and counseling also offered.

\section{Discussion}

The above presented cases showing common pertinent pattern of presentation such as insidious onset of illness, predominance of somatic complains that were misdiagnosed and mismanaged, involvement of both genders, the change in presentation from the initial illness phase, Preoccupation with abnormal physical sensations i.e. secretions from the body, the strong conviction with their beliefs, lack of insight to correct that abnormal belief. Nevertheless their lives were revolving around those false perceptions with intense distress and sociooccupational impairment, Absence of other physical comorbid and clinical signs of other psychiatric disorders (Depression, Obsessive Compulsive Disorder-OCD and mania) as per diagnostic criteria.

The insidious course, lack of insight, strong conviction to the belief, behavioral alteration and socio-occupational impairment supported to consider for psychotic disorder i.e., Schizophrenia. The other studies in comparison to presented cases also mirrored most of the findings such as chronic insidious course (Chang, 2014), the predominant oral symptoms presentation in males, the late onset of presentation in females (>40yrs) (Takahash, et al., 2013).There is also a frequent presentation of chronic medically unexplained physical symptoms and misdiagnosis as somatoform disorder (Rohircht, et al., 2010). Due to misdiagnosis and neglected care these are usually lately diagnosed as in presented cases (Rohircht, et al., 2010). The commonly identified hurdles in the early diagnosis are lack of knowledge and awareness due to non-inclusion of this diagnosis of cenesthopathic schizophrenia as a separate entity as well as appropriate assessment tools are not easily available to facilitate or confirm the diagnosis (Jenkins, et al., 2007 \& Stanghellini, et al., 2014).The delayed diagnosis is usually associated with poor prognosis. Early identification and treatment with second generation antipsychotics, electroconvulsive therapy and antidepressants in indicated cases predicts good prognosis (Takahash, et al., 2013). Almost half of the cases found to respond well on antipsychotics (Rohircht, et al., 2010).

\section{Recommendations}

- First of all it is essential to include this diagnosis as separate entity of schizophrenia in the standard classification systems

- Need to educate the health professional to early recognize these cases for better prognosis

- Improvement in making the health care services more accessible

- Appropriate assessment tools must be easily available both for clinics and research.

- Future research is needed to understand the psychophysiological mechanisms and to establish better evidence of treatment.

\section{References}

- Blom, J. D., \& Sommer, I. E. (2012). Hallucinations of bodily sensation. 
In Hallucinations (pp.

157-169).

Springer New York.

- Chang W. (2014) Cenesthopathic schizophrenia (case conference TYPC Taiwan 20141227)

- Grignon, S., Stavro, K., \& Potvin, S. (2014). Could schizophrenia be a refractory condition to central pain sensitization? In Mental Health and Pain (pp. 157-171). Springer Paris.

- Jenkins, G., \& Röhricht, F. (2007). From cenesthesias to cenesthopathic schizophrenia: a historical and phenomenological review. Psychopathology, 40(5), 361368.

- Rajender, G., Kanwal, K., Rathore, D. M., \& Chaudhary, D. (2009). Study of cenesthesias and body image aberration in schizophrenia. Indian journal of psychiatry, 51(3), 195.195-98.

- Röhricht, F., Gudi, A., \& LewisFernández, R. (2010). Medically unexplained physical symptoms masking (cenesthopathic) schizophrenia: a case series. Journal of Psychiatric Practice ${ }^{\circledR}, 16(4)$, 258-264.

- Stanghellini, G., Ballerini, M., Blasi, S., Mancini, M., Presenza, S., Raballo, A., \& Cutting, J. (2014). The bodily self: a qualitative study of abnormal bodily phenomena in persons with schizophrenia. Comprehensive psychiatry, 55(7), 1703-1711.

- Stanghellini, G., Ballerini, M., Fusar Poli, P., \& Cutting, J. (2012). Abnormal bodily experiences may be a marker of early schizophrenia? Current Pharmaceutical Design, 18(4), 392-398.

- Takahashi, T., Fuke, T., Washizuka, S., Hanihara, T., \& Amano, N. (2013). A review of recent case reports of cenesthopathy in Japan. Psychogeriatrics, 13(3), 196-198. 